\title{
Shaggy, the Homolog of Glycogen Synthase Kinase 3, Controls Neuromuscular Junction Growth in Drosophila
}

\author{
Bénédicte Franco, ${ }^{1}$ Laurent Bogdanik, ${ }^{1}$ Yves Bobinnec, ${ }^{2}$ Alain Debec, ${ }^{2}$ Joël Bockaert, ${ }^{1}$ Marie-Laure Parmentier, ${ }^{1}$ and \\ Yves Grau ${ }^{1}$ \\ ${ }^{1}$ Laboratoire de Génomique Fonctionnelle, Unité Propre de Recherche Centre National de la Recherche Scientifique 2580, 34094 Montpellier Cedex 5, \\ France, and ²Biologie du Développement, Unité Mixte de Recherche 7009, Observatoire Océanologique, 06230 Villefranche sur Mer, France
}

A protein-trap screen using the Drosophila neuromuscular junction (NMJ) as a model synapse was performed to identify genes that control synaptic structure or plasticity. We found that Shaggy (Sgg), the Drosophila homolog of the mammalian glycogen synthase kinases $3 \alpha$ and $\beta$, two serine-threonine kinases, was concentrated at this synapse. Using various combinations of mutant alleles of shaggy, we found that Shaggy negatively controlled the NMJ growth. Moreover, tissue-specific expression of a dominant-negative Sgg indicated that this kinase is required in the motoneuron, but not in the muscle, to control NMJ growth. Finally, we show that Sgg controlled the microtubule cytoskeleton dynamics in the motoneuron and that Futsch, a microtubule-associated protein, was required for Shaggy function on synaptic growth.

Key words: motoneuron; morphological plasticity; microtubule loop; microtubule-associated protein; glycogen synthase kinase; synaptic plasticity

\section{Introduction}

Once established, synaptic connections can be modified throughout animal life. Physiological tuning of synapses may be accompanied by morphological changes of these synapses. Such a synaptic plasticity is thought to be the cellular basis of information processing and storage in the brain (Muller et al., 2002).

To identify the molecular mechanisms controlling synaptic structure and plasticity, we undertook a genetic approach in Drosophila melanogaster, focusing on the larval neuromuscular junction (NMJ). This synapse is functional at the end of embryogenesis and is required for the contraction of the body-wall muscles, leading to larval hatching and larval locomotion. This synapse shares important features with central excitatory synapses of the mammalian brain: it is a glutamatergic synapse containing ionotropic glutamate receptors, the postsynaptic density protein Discs-Large (DLG; homologous to PSD-95) and other proteins also found in mammalian central synapses (Gramates and Budnik, 1999; Broadie and Richmond, 2002). Finally, the Drosophila NMJ is a dynamic structure with morphological plasticity that depends on its activity as well as on the size of the target muscles. For example, during larval development, there is a 10 -fold in-

Received Nov. 12, 2003; revised June 1, 2004; accepted June 1, 2004.

This work was supported by the Centre National de la Recherche Scientifique, Institut National de la Santé et de la Recherche Médicale, and the French Ministry of Research and Technology. We thank V. Budnik, E. Buchner, G. Davis, P. Heitzler, C. Klämbt, C. O'Kane, C. Schuster, and D. Woods for providing fly stocks or antibodies. We thank M. Bourouis and V. Homburger for discussions and J.-P.Pin, F. Maschat, J.-M. Dura, and C. Mitri for critical reading of this manuscript.

Correspondence should be addressed to Marie-Laure Parmentier, Unité Propre de Recherche Centre National de la Recherche Scientifique 2580, 141 rue de la Cardonille 34094 Montpellier Cedex 5, France. E-mail: mlparmentier@ccipe.cnrs.fr.

D0I:10.1523/JNEUROSCI.1580-04.2004

Copyright $\odot 2004$ Society for Neuroscience $\quad$ 0270-6474/04/246573-05\$15.00/0 crease in the number of synaptic boutons in correlation with the growth of the target muscle (Schuster et al., 1996a). Furthermore, the hyperactive eag $S h$ mutants show a twofold increase in the number of boutons compared with wild-type larvae (Schuster et al., 1996b).

Protein kinases are responsible for the phosphorylation of proteins, a major post-translational modification involved in numerous signaling and regulation pathways. Protein kinases represent one of the largest families of proteins, all possessing a common catalytic core. There are $>450$ kinases in the human genome and $\sim 255$ kinases in the Drosophila genome. Many of these kinases play a role from early development onward, and mutants for the corresponding genes die during embryonic development. Thus, it is difficult to genetically screen for the role of such proteins in synaptic structure and plasticity at late stages of development when the synapses are established.

Here, we used a protein-trap screen (Morin et al., 2001) where a green fluorescent protein (GFP) exon is inserted within genes, leading to fusion fluorescent proteins. We selected a line where this exon is inserted in the shaggy ( $\mathrm{sgg}$ ) gene. Shaggy is the Drosophila homolog of mammalian glycogen synthase kinase (GSK) 3. We observed that Sgg is concentrated in the presynaptic side of the NMJ. Using a hypomorph mutation of $s g g$, we discovered that this kinase negatively controls NMJ growth. Motoneuronspecific expression of a dominant-negative isoform of Sgg revealed that this kinase is required presynaptically for this NMJ growth control. Finally, inhibition of Sgg leads to an increase in the number of microtubule loops, suggesting that part of the growth control of the NMJ by Sgg occurs via changes in the microtubule cytoskeleton dynamics. This is supported by epistasis experiments between $s g g$ and futsch, a gene encoding a 
microtubule-associated protein (MAP), showing that futsch is indeed downstream of $s g g$.

\section{Materials and Methods}

Fly stocks. The $s g g^{K 22}$ mutant and the $s g g$ mini10 transgene were obtained from P. Heitzler (Institut de Génétique et de Biologie Moléculaire et Cellulaire, Illkirch, France). The sgg mini10 construct carries the cDNA sequence of the SGG10 isoform fused to genomic untranslated region sequences of $\mathrm{sgg}$ (Ruel et al., 1993). Upstream activating sequence (UAS) $-s g g^{D N}$ stocks (on chromosomes 2 and 3) and deficiency $D f(1) 64 j 4$ were obtained from the Bloomington Stock Center (Bloomington, IN). We used the Gal4 drivers elavC155 and myosin heavy chain (MHC)-Gal4 (C. Goodman, University of California, Berkeley, CA), BG380 (V. Budnik, University of Massachusetts Medical School, Worcester, MA), and OK6 (C. O'Kane,
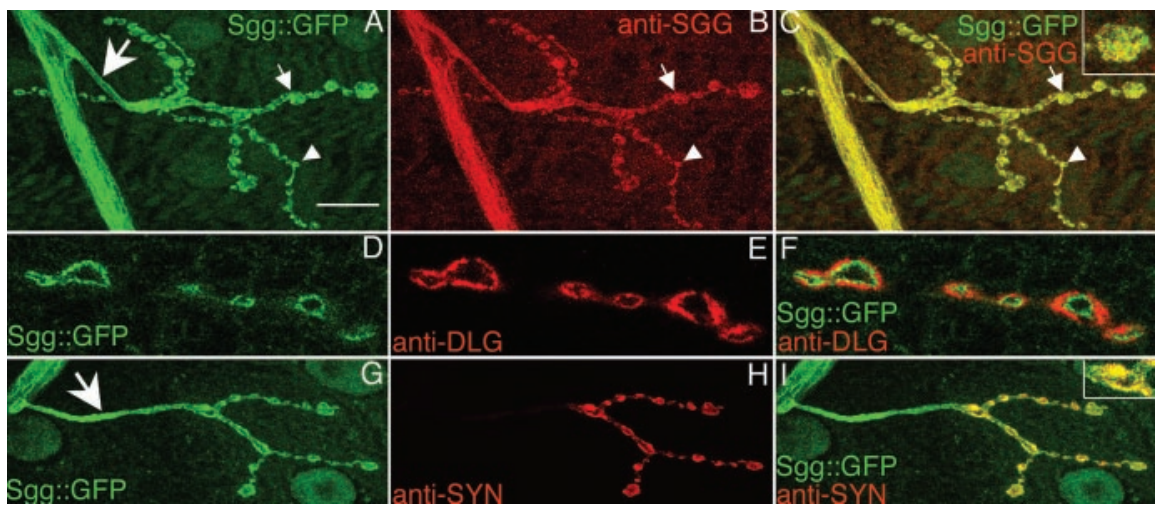

Figure 1. Synaptic localization of Sgg::GFP and Shaggy. GFP fluorescence $(A, D, G)$ and anti-SGG $(B)$, anti-DLG $(E)$, and anti-Synapsin (SYN) $(H)$ immunostainings on 105-1 protein trap line. All NMJs shown are located on muscle 4, but the same results could be observed on any other body-wall muscle. Color merges are shown $(C, F, I)$, with higher magnification of one varicosity in $C$ and $I$. Small arrows indicate type $\mathrm{Ib}$ boutons, and arrowheads indicate type $\mathrm{Is}$ boutons. Large arrows indicate the nerve branch. Scale bars: $A-C, G-I, 20 \mu \mathrm{m} ; D-F, 10 \mu \mathrm{m}$.

University of Cambridge, Cambridge, UK). We

obtained stronger phenotypes with the chromosome 2 UAS-sgg $g^{D N}$ (see Fig. $3 C$ ) compared with chromosome 3 UAS-sgg ${ }^{D N}$ (see Fig. $4 D$ ). The futsch $^{N 94}$ and futsch ${ }^{K 68}$ alleles were obtained from C. Klämbt (Universität of Münster, Münster, Germany) and G. Davis (University of California, San Francisco, CA). All stocks were reared at $25^{\circ} \mathrm{C}$, except crosses with the UAS$s g g^{D N}$ that were reared at $29^{\circ} \mathrm{C}$ during larval stages and larvae used for Figure $4 D$. Wild-type flies were Canton S (CS) or $y w^{C S}$.

To perform epistasis experiments between futsch and sgg, we constructed a (OK6-Gal4; UAS-sgg $\left.{ }^{D N}\right) /$ SM5:TM6B stock, which was crossed with futsch mutant females to obtain futsch ${ }^{N 94}$ or futsch ${ }^{K 68} / \mathrm{Y}$; OK6/+; UAS-sgg ${ }^{D N} /+$ larvae.

Immunocytochemistry and confocal microscopy. Wandering third instar larvae were dissected in $4 \%$ paraformaldehyde and $1 \times$ PBS. The following antibodies were used: mouse monoclonal anti-GSK-3 $\beta$, raised against an epitope conserved in Sgg (1:100; Upstate Biotechnology, Lake Placid, NY), guinea-pig anti-DLG (1:1000; D. Woods, University of California, Irvine, CA), mouse monoclonal anti-Synapsin (1:100; E. Buchner, Universität Würzburg, Würzburg, Germany), mouse monoclonal anti-cystein string protein (CSP) (1:10, K. Zinsmaier, University of Arizona, Tucson, AZ), mouse monoclonal anti-Futsch (22C10, 1:100) (Roos et al., 2000), and rabbit polyclonal anti-horse radish peroxydase (HRP) (1:1000; Sigma, St. Louis, MO). 22C10 was obtained from the Developmental Studies Hybridoma Bank (University of Iowa, Iowa City, IA). Fluorescent-conjugated antibodies were from Jackson ImmunoResearch (West Grove, PA) and Molecular Probes (Eugene, OR). Confocal images were acquired using a Bio-Rad (Hercules, CA) MRC 1024 laser-scanning confocal microscope (Centre Régional d'Imagerie Cellulaire, Montpellier, France).

Quantification. Synaptic boutons (only type Ib) on muscles 6/7 of the A3 segment were counted from HRP-stained preparations on a wide-field fluorescence microscope (Zeiss Axiophot; Zeiss, Thornwood, NY) at $100 \times$ magnification. A picture of the inner surface of muscle $6 / 7$ was taken at $20 \times$ magnification to quantify the muscle area. The normalized bouton number was calculated by dividing bouton numbers by muscle surface area (Schuster et al., 1996a). Data were expressed as a percentage of the control of each experiment. We observed no major differences in muscle size between the genotypes. Hence, rough bouton number quantification yielded the same phenotypes as normalized bouton number. To correct for variations in total bouton number, we quantified the amount of satellite boutons as a percentage of the mean total bouton number for each genotype. Statistical analysis was performed using a two-tailed Student's $t$ test.

\section{Results}

\section{SGG is enriched in the presynaptic side at the NMJ}

To visualize proteins concentrated at the NMJ, we took advantage of a GFP transposon-based screen, in which a GFP sequence engineered as an exon is randomly inserted in genes (Morin et al.,
2001). Insertions of the GFP exon in frame with an expressed gene were recovered by screening larvae for fluorescence. We selected lines that presented a clear fluorescence at the third instar larvae NMJs. In line 105-1, the GFP exon was inserted in the second intron of the $s g g$ gene (position 8665 of scaffold AE003425.3), thus producing an Sgg::GFP fusion protein. We confirmed the in-frame insertion of the GFP sequence by $5^{\prime}$ and $3^{\prime}$ rapid amplification of cDNA ends experiments (data not shown). Null mutations of sgg are lethal at the embryonic stage or the first instar larval stage (Ruel et al., 1993). Here, the transgenic line was homozygous viable and fertile, suggesting that the Sgg::GFP fusion protein was functional. This transgenic line presented a clear fluorescence at the NMJs of all body-wall muscles. Both type Ib and Is synaptic boutons, which correspond to different glutamatergic motoneurons innervating the muscles, expressed Sgg::GFP (Fig. 1A). At the NMJ, all of the GFP signal overlapped with an anti-Sgg immunostaining, indicating that the GFP signal indeed corresponded to tagged Shaggy proteins (Fig. $1 A-C)$. We obtained qualitatively the same anti-Sgg signal on wild-type CS flies, indicating that Sgg::GFP localization was the same as the wild-type Sgg localization (data not shown). Comparison of Sgg::GFP fluorescence with the postsynaptic marker DLG (Lahey et al., 1994) revealed that Sgg::GFP was not localized in the postsynaptic side but was presynaptic (Fig. $1 D-F$ ). We confirmed this presynaptic localization by observing that Sgg::GFP colocalized with the vesicle marker Synapsin (Klagges et al., 1996) (Fig. 1G-I). Moreover, we also detected some clear Sgg::GFP signal in axonal branches (Fig. $1 A, G$, arrows). Altogether, these results show that Sgg is enriched in the presynaptic side of the NMJ.

\section{Mutation in sgg alters the growth of the NMJ}

To examine sgg function during the development of the NMJ, we used the hypomorph mutation $s g g^{K 22}$. This mutation is viable until the third instar larval stage. We did not detect any Sgg immunoreactivity at the NMJs of $s g g^{K 22}$ third instar larvae (Fig. $2 A-D)$ nor any Sgg product on Western blot from third instar larva protein extracts (data not shown). In all of our experiments using sgg mutants, we chose crawling larvae, indicating that the NMJs were functional.

During larval development, there is constant adaptation of the NMJ size to the increase in muscle size. This includes an increase in the number and size of synaptic boutons as well as in the 


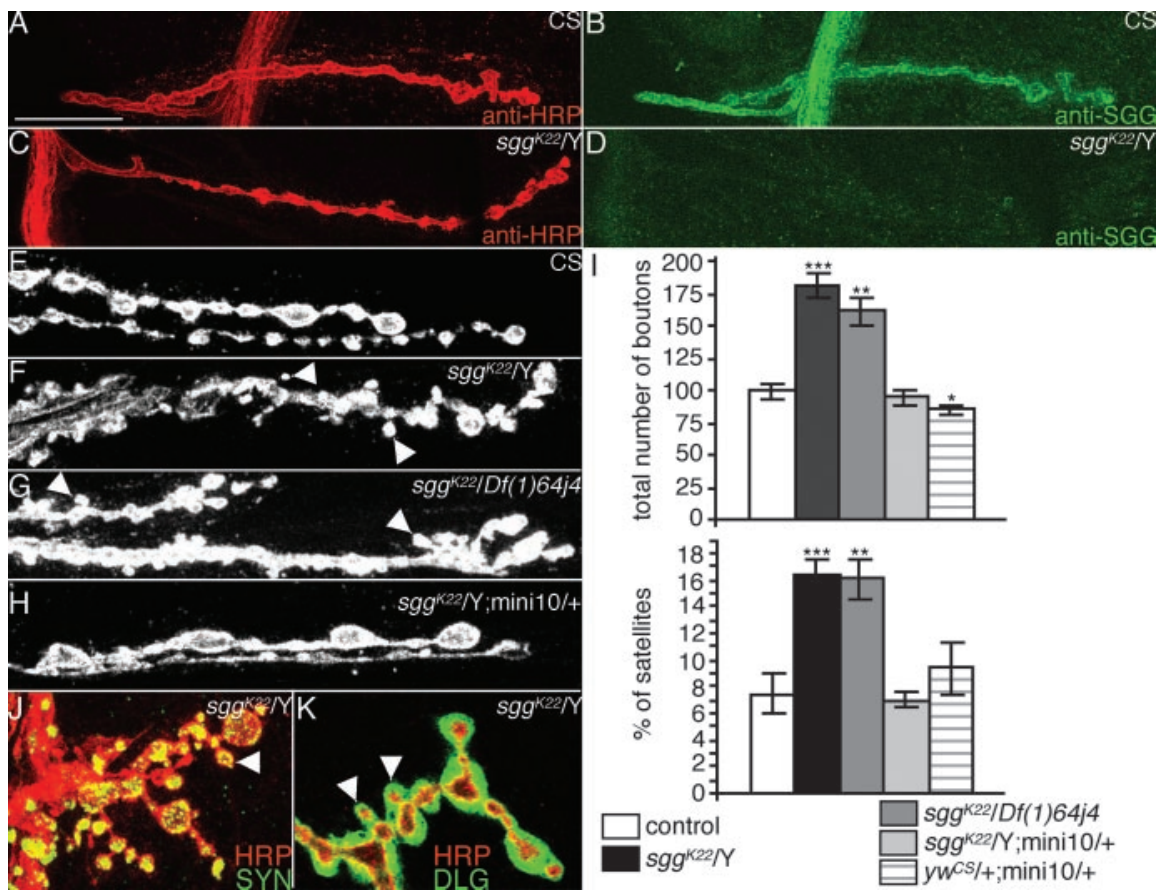

Figure 2. Morphological phenotype of sgg mutants. $A-D, A$ nti-HRP $(A, C)$ and anti-SGG $(B, D)$ immunostainings on a $C S$ male larva $(A, B)$ or sgg ${ }^{K 22} / Y$ larva $(C, D)$. E-H, Illustration of the overgrowth phenotype in sgg ${ }^{K 22} / Y(F)$ or sgg $g^{K 22} / D f(1) 64 j 4(G)$ mutant larvae. This phenotype is rescued to the wild-type range $(E)$ by the addition of an sgg minigene (mini10) in the genome of $s g g^{K 22}$ mutants $(H)$. The NMJs of muscle $6 / 7$ are visualized with an anti-HRP staining. $I$, Quantification of the total number of $I b$ boutons and the percentage of satellite boutons at muscles 6/7 NMJs of CS male $(n=12), s_{g} g^{K 22} / \mathrm{Y}(n=25), \operatorname{sgg}^{K 22} / \mathrm{Df}(1) 64 j 4(n=29)$, $s g g^{K 22} / Y$; mini10/+ $(n=14)$, and $y w^{C S} /+; \operatorname{mini10} /+(n=13)$ larvae. Statistical comparisons to the CS control: * $p<0.05$; ${ }^{* *} p<0.01 ;{ }^{* * *} p<0.001$. J, Anti-SYN (green) and anti-HRP (red) double staining on an $s g g^{K 22} / Y$ muscle 13 NMJ. K, Anti-DLG (green) and anti-HRP (red) double staining on an $\mathrm{sg}^{K 22} / \mathrm{Y}$ muscle $13 \mathrm{NMJ}$. In all images, arrowheads indicate satellite boutons. Scale bars: $A-D, 20 \mu \mathrm{m} ; F-H, 11 \mu \mathrm{m} ; J, K, 14 \mu \mathrm{m}$.

number of active zones (Schuster et al., 1996a; Lnenicka and Keshishian, 2000). We examined synaptic morphology by labeling presynaptic terminals using anti-HRP, an insect neuronal marker. Analysis of NMJs in $s g g^{K 22}$ mutants showed that the loss of $s g g$ function had clear consequences for the growth of the synapse. Indeed, NMJ expansion was dramatically increased, resulting in an $80 \%$ increase in the number of type Ib boutons on muscle 6/7 in $s g g^{K 22}$ mutant larvae compared with wild-type controls (Fig. $2 E-I)\left(100.0 \pm 5.8\right.$ for CS; $181.4 \pm 9.7$ for $\left.s g g^{K 22} / Y\right)$. To ensure that this phenotype was caused by shaggy loss of function and not to a second site mutation, we used the deficiency $D f(1) 64 j 4$ known to delete the entire sgg gene (Ruel et al., 1993). $s g g^{K 22} / D f(1) 64 j 4$ larvae also displayed a $60 \%$ increase in bouton number (161.9 \pm 10.3$)$. Interestingly, in both sgg loss-offunction genotypes, the increase in bouton number was accompanied by a twofold increase in the number of "satellite" boutons (Torroja et al., 1999) [7.4 \pm 1.5 for CS; $16.5 \pm 1.1$ for $\mathrm{sgg}^{\mathrm{K} 22} / \mathrm{Y}$; $16.1 \pm 1.5$ for $\mathrm{sgg}^{K 22} / D f(1) 64 j 4$ ] (i.e., boutons budding off from a bouton present in the axis of the branch, or boutons budding off from neuronal connections between two boutons) (Fig. $2 F-I$ ). Both phenotypes of $s g g^{K 22}$ mutant larvae were rescued to wildtype range by adding one copy of a wild-type sgg minigene (mini10) in the genome (94.9 \pm 5.4 for type Ib boutons; $7.0 \pm 0.6$ for satellite boutons) (Fig. $2 \mathrm{H}, I$ ). This demonstrates that these phenotypes were attributable to the loss of shaggy function.

Because sgg loss-of-function mutants displayed an overdeveloped NMJ, we would expect to observe the opposite phenotype in the presence of a gain of $s g g$ function. We tested this hypothesis by quantifying the number of synaptic boutons in larvae possessing, in addition to the wild-type sgg gene on the $\mathrm{X}$ chromosome, an additional autosomal copy of sgg. In these larvae, the total number of Ib boutons $(85.0 \pm 4.3)$ was decreased compared with the control larvae, whereas the percentage of satellite boutons $(9.4 \pm 1.9)$ was not significantly modified. These data show that a dose increase of $s g g$ is sufficient to slightly shrink the NMJ.

To determine whether all boutons in sgg mutant synapses had normal features of synaptic boutons, we studied the localization of presynaptic and postsynaptic markers. Analysis of presynaptic synapsin (Fig. 2J) and CSP (data not shown) revealed that each bouton, and notably the satellite ones, was immunopositive for these markers. Similarly, we found that all boutons were surrounded by postsynaptic DLG immunoreactivity (Fig. $2 \mathrm{~K}$ ). Thus, the mutant boutons, showing a wild-type localization of some elements of the presynaptic and postsynaptic apparatus, are probably functional. Altogether, these results show that Sgg has an inhibitory role in expanding the NMJ but may not play an important role in bouton differentiation.

\section{Presynaptic Sgg controls the NMJ growth}

Anti-Sgg immunostaining as well as Sgg::GFP fluorescence were concentrated in the presynaptic side, suggesting a role of the presynaptic Sgg in the phenotypes described above. To examine the function of presynaptic Sgg, we used a dominant-negative version of the Sgg10 isoform (Bourouis, 2002). The mutation introduced in the coding sequence is the substitution A81T leading to a "kinase-null" enzyme. We overexpressed this transgene $\left(s g g^{D N}\right)$ under the control of different presynaptic (or postsynaptic) Gal4 drivers and quantified the bouton number. For presynaptic expression, we used the panneuronal elav-Gal4 driver as well as the BG380-Gal4 and OK6-Gal4 drivers, which have patterns of expression more restricted to motoneurons. For postsynaptic expression, we used the MHC-Gal4 driver, which drives expression in all body-wall muscles, as checked with UAS-mCD8GFP (data not shown). In all genotypes where Sgg ${ }^{\mathrm{DN}}$ is expressed presynaptically, we observed a $50-100 \%$ increase in the number of synaptic boutons (Fig. 3A-C) $(100.0 \pm 5.5$ for CS; $157.4 \pm 6.4$ for BG380-Gal4; $164.9 \pm 9.8$ for elav-Gal4; $212.8 \pm 18.3$ for OK6-Gal4). Interestingly, the number of boutons did not change when the dominant-negative construct was expressed postsynaptically (Fig. 3C) (92.6 \pm 4.4 for MHC-Gal4). The results were similar when we considered the satellite boutons: presynaptic inhibition of Sgg led to a twofold to threefold increase in the percentage of satellite boutons $(8.0 \pm 0.9$ for CS; $12.9 \pm 1.3$ for BG380-Gal4; $15.8 \pm 1.5$ for elav-Gal4; $20.2 \pm 1.5$ for OK6-Gal4), whereas postsynaptic inhibition of Sgg led to no changes (Fig. 3C) (7.5 \pm 0.8 for MHC-Gal4).

Again, immunostainings against the presynaptic markers CSP, Synapsin, and the postsynaptic marker DLG revealed that all boutons were immunoreactive for these markers, suggesting that they are all functional (data not shown). Altogether, our 


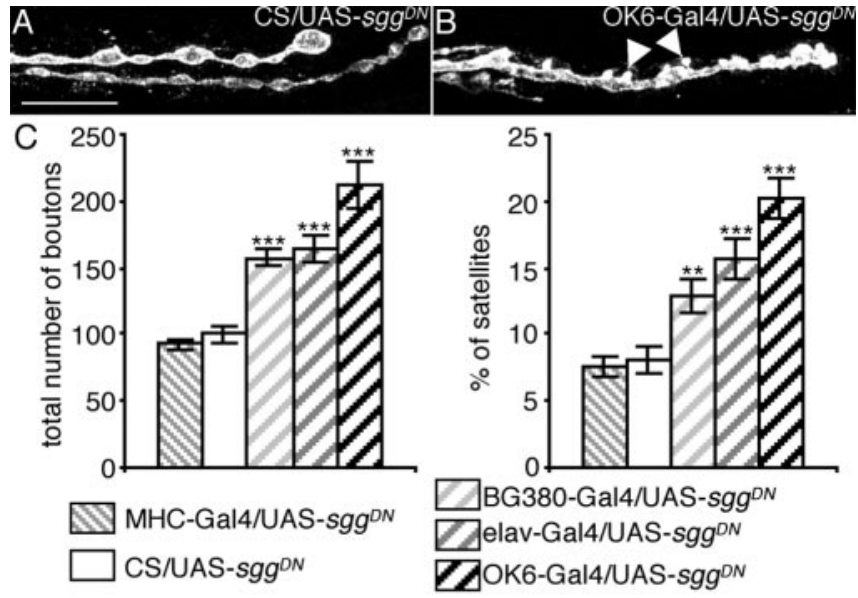

Figure 3. Overexpression of a dominant-negative $\mathrm{Sgg}$ with different $\mathrm{Gal} 4 \mathrm{drivers.} A, B, \mathrm{Illus}-$ tration of the overgrowth phenotype when $\mathrm{Sgg}{ }^{\mathrm{DN}}$ is expressed presynaptically: anti-HRP staining of a muscle 6/7 NMJ of a control female larva (CS/UAS-SgD $\left.{ }^{D N}\right)(A)$ or a female overexpressing Sgg ${ }^{D N}$ presynaptically (0K6-Gal4/UAS-sgg $g^{D N}$ ( $(B)$. Arrowheads indicate satellite boutons. Scale bar, $11 \mu \mathrm{m}$. C, Quantification of the total bouton number and the percentage of satellite boutons of control and $\mathrm{Sgg}^{\mathrm{DN}}$ overexpressing flies. $n=25,26,18,31$, and 14 for crosses with CS, MHC-Gal4, BG380-Gal4, elav-Gal4, and 0K6-Gal4, respectively. ${ }^{* *} p<0.01$; ${ }^{* * *} p<0.001$.

results show that presynaptic but not postsynaptic kinase activity of Sgg is required for normal synaptic growth.

Sgg controls the microtubule cytoskeleton dynamics

One feature of the Sgg inhibition phenotype was the increase in the percentage of satellite boutons. No such increase is found in other mutants displaying an NMJ overgrowth phenotype, like the eag Sh hyperactive mutants (Budnik et al., 1990; Torroja et al., 1999). Satellite boutons can be considered potential ramifications because they are not in line with the other boutons. Ramifications of the NMJ have recently been shown to occur when the microtubule cytoskeleton forms a loop within a bouton (Roos et al., 2000). We wondered whether the increase in satellite boutons observed in sgg loss-of-function conditions was related to modifications in the microtubule cytoskeleton dynamics. We therefore performed stainings directed against the Drosophila MAP1B homolog, Futsch, and quantified the number of microtubule loops when the dominant-negative form of Sgg was expressed in the motoneurons or in the muscle. There were effectively more loops when the percentage of satellite boutons increased in larvae expressing Sgg ${ }^{\text {DN }}$ presynaptically (Fig. $\left.4 A-C\right)$ (1.6 \pm 0.2 for CS; $3.8 \pm 0.6$ for BG380-Gal4; $3.0 \pm 0.3$ for elav-Gal4; $5.5 \pm 0.4$ for OK6-Gal4). In larvae expressing Sgg ${ }^{\mathrm{DN}}$ postsynaptically, the amount of microtubule loops ( $1.0 \pm 0.3$ for MHC-Gal4), like the percentage of satellite boutons, remained unchanged. In Figure $4 B$, we show that many loops were localized in satellite boutons (arrows). Altogether, these results show that Sgg controls the dynamics of the microtubule cytoskeleton, such that an inhibition of the kinase leads to an increase in the number of microtubule loops, which is possibly the cause of the increase in the amount of satellite boutons.

\section{Futsch is downstream of Shaggy}

To find out whether microtubule effectors are actually downstream of shaggy, we performed epistasis experiments. We used two futsch hypomorph loss-of-function mutations, futsch ${ }^{N 94}$ and futsch $^{K 68}$, which respectively display a partially or strongly disorganized microtubule cytoskeleton. Moreover, microtubule loop
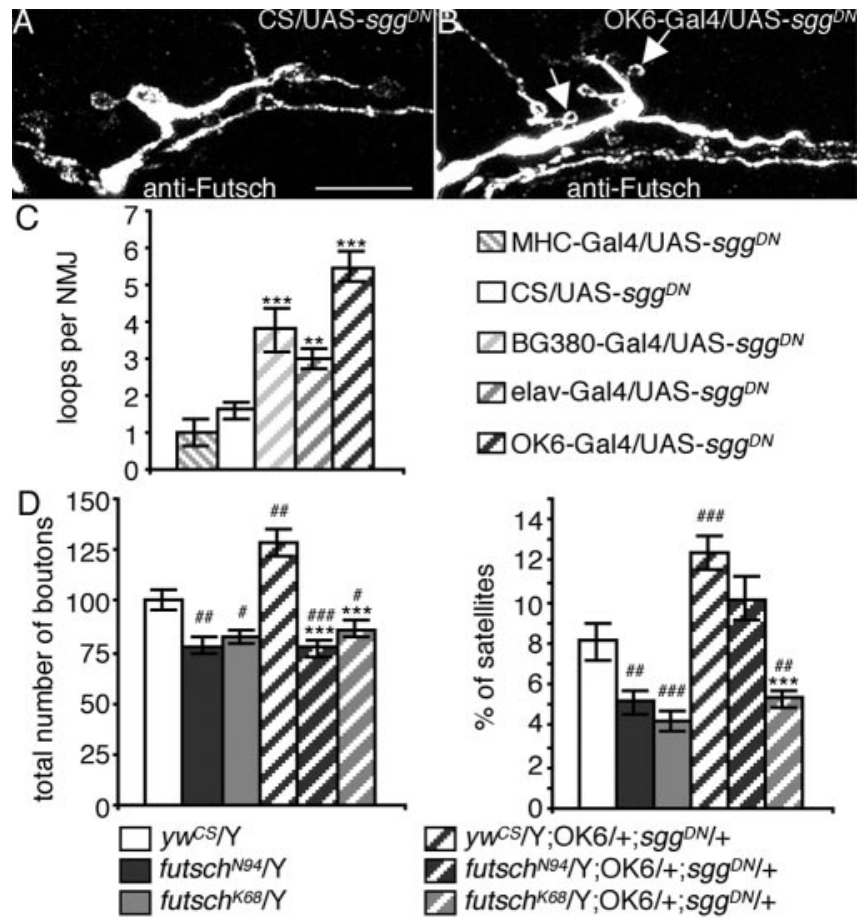

Figure 4. Effect of $\mathrm{Sgg}{ }^{\mathrm{DN}}$ overexpression on microtubule dynamics and genetic interaction with futsch. $A, B$, Illustration of the increase in the number of microtubule loops when $\mathrm{Sgg}^{\mathrm{DN}}$ is expressed presynaptically: anti-Futsch staining of a muscle $13 \mathrm{NMJ}$ on a control female larva $\left(C S / U A S-s g g^{D N}\right)(A)$ or a female overexpressing Sgg ${ }^{\text {DN }}$ presynaptically (OK6-Gal4/UAS-sgg ${ }^{D N}$ ) (B). Arrows indicate loops within satellite boutons. Scale bar, $10 \mu \mathrm{m}$. C, Quantification of the number of microtubule loops in control larvae versus larvae expressing the $\mathrm{Sgg}{ }^{\mathrm{DN}}$ protein. $n=$ $15,9,9,15$, and 6 for UAS-sgg ${ }^{D N}$ crosses with CS, MHC-Gal4, BG380-Gal4, elav-Gal4, and OK6Gal4. ${ }^{* *} p<0.01{ }^{* * *} p<0.001$. D, Genetic interaction between $\mathrm{Sgg}{ }^{\mathrm{DN}}$ expressed presynaptically and futsch ${ }^{N 94}$ or futsch ${ }^{K 68}$. Quantification of boutons number and percentage of satellite boutons of larvae expressing Sgg ${ }^{\mathrm{DN}}$ in the presynapse in a wild-type, futsch ${ }^{\mathrm{N} 94}$, and futsch ${ }^{\mathrm{K} 68}$ genetic background. $n=29$ for $y w^{\mathrm{CS}} / \mathrm{Y}, 22$ for futsch ${ }^{\mathrm{N94}} / \mathrm{Y}$ and futsch ${ }^{\mathrm{K} 68} / \mathrm{Y}, 28$ for $y w^{\mathrm{CS}} / \mathrm{Y} ; 0 \mathrm{~K} 6 /$ $+; s g g^{D N} /+, 30$ for futsch ${ }^{N 94} / Y ; 0 K 6 /+; s g g^{D N} /+$, and 22 for futsch ${ }^{K 68} / \mathrm{Y} ; 0 \mathrm{~K} 6 /+; s g g^{D N} /+$. ${ }^{* * *} p<0.001$ for statistical significance versus $y w^{C S} / \mathrm{Y} ; 0 \mathrm{~K} 6 /+;$ sgg $^{D N} /+{ }^{*} p<0.05 ;{ }^{\# \#} p<$ $0.01 ; \# \#<0.001$ for statistical significance versus $y w^{C S} / Y$.

formation is absent in both mutants (Roos et al., 2000). We verified that the two futsch mutants displayed a 20\% decreased number of synaptic boutons compared with CS larvae, as shown by Roos et al. (2000) (Fig. 4D) (100.0 \pm 5.0 for $y w^{C S}$; $78.1 \pm 4.6$ for futsch $^{N 94} ; 82.5 \pm 3.9$ for futsch $\left.{ }^{K 68}\right)$. We also detected a significant decrease in the percentage of satellite boutons in these mutants (Fig. 4D) (8.1 \pm 0.9 for $y w^{C S} ; 5.2 \pm 0.6$ for $f u t s c h^{N 94} ; 4.2 \pm 0.5$ for futsch $\left.^{K 68}\right)$. These phenotypes are opposite of those observed in $s g g$ loss-of-function conditions. Hence, if the NMJ overgrowth observed in $s g g$ mutants requires intact microtubule effectors, we should not see this phenotype when the microtubule cytoskeleton is affected. To test this hypothesis, we presynaptically overexpressed the dominant-negative form of Sgg in wild-type or futsch mutant background (see Materials and Methods). Although NMJs of larvae expressing Sgg ${ }^{\mathrm{DN}}$ presynaptically in a wild-type background presented a $30 \%$ increase of bouton number $(128.5 \pm 6.8)$, NMJs of larvae expressing $\mathrm{Sgg}^{\mathrm{DN}}$ in either futsch $^{N 94}$ or futsch ${ }^{K 68}$ background did not display any increase in bouton number $(76.7 \pm 4.4$ and $86.4 \pm 3.8$, respectively) but displayed the futsch phenotype (Fig. 4D). The situation was similar when we looked at the satellite bouton phenotype (Fig. 4D). Larvae expressing Sgg ${ }^{\mathrm{DN}}$ in the futsch ${ }^{K 68}$ background displayed the futsch ${ }^{K 68}$ phenotype $(5.3 \pm 0.3)$. Interestingly, the phenotype was intermediate in the futsch ${ }^{N 94}$ background (10.2 \pm 1.1$)$. This 
may be attributable to the remaining Futsch protein detected in these mutants. We also performed Futsch immunostaining of NMJs of futsch ${ }^{N 94}$ larvae either expressing or not expressing the Sgg ${ }^{\mathrm{DN}}$ transgene. In both cases, Futsch protein was diffusely localized in the synaptic boutons, indicating that the inhibition of Sgg does not affect the localization of this mutant Futsch protein (data not shown).

Altogether, our data demonstrate that the NMJ overgrowth observed when Sgg is inhibited presynaptically requires the microtubule-associated protein Futsch.

\section{Discussion}

The gene sgg is known to play an important role in embryo morphogenesis and cell fate determination (Ruel et al., 1993). Here, our screen let us find that the kinase encoded by this gene was highly concentrated at larval motoneuron terminals. This suggested a role for this protein in these differentiated neurons. In accordance with this hypothesis, we showed, using the mutant $s g g^{K 22}$ and a dominant-negative construct of Sgg, that this kinase negatively controlled the NMJ growth during the larval stages. These data are the first to reveal a role of this kinase in the growth of a differentiated and functional synapse. Using different presynaptic and postsynaptic markers, we could not detect any obvious defect in synapse differentiation. However, the $s g g^{K 22}$ allele is not a null allele. Thus, we cannot exclude that shaggy plays other roles than growth control at the NMJ.

The function of GSK-3 $\beta$ in neuronal development, and notably in synapse differentiation had been studied previously on neuronal cultures using lithium chloride $(\mathrm{LiCl})$ as a GSK- $3 \beta$ inhibitor (Lucas and Salinas, 1997; Hall et al., 2000). These authors observed more synapsin-positive clusters along the axons in the presence of LiCl (Hall et al., 2000). This last observation was interpreted as an increase in accumulation of synapsin at the synapses (i.e., a modification in the differentiation of synapses). However, we can interpret this result as an increase in the number of synapses. This in vitro result would then be in accordance with our in vivo data at the Drosophila NMJ, where inhibition of Shaggy increases the number of synaptic boutons.

Targeted expression of Sgg ${ }^{\mathrm{DN}}$ showed that the function of Shaggy on the growth of the NMJ was required presynaptically and that it requires the kinase function of Sgg. Some major presynaptic targets of GSK-3 $\beta$ known in vertebrates are microtubule-associated proteins like tau, MAP1B, and MAP2 (Cohen and Frame, 2001). Here, we show that when we inhibit Shaggy in the motoneuron, there is an increase in the number of microtubule loops. This change in the dynamics of the microtubule skeleton suggests that some microtubule-associated proteins like the Drosophila tau homolog or the MAP1B homolog, Futsch, may also be substrates of Shaggy. Of interest, we could identify consensus sites for GSK-3 like phosphorylation $[\mathrm{SXXX}(\mathrm{ST})(\mathrm{PR}) \mathrm{XXS}]$ in the sequence of these proteins (96 for Futsch; 1 for tau) but do not have evidence yet that these proteins are phosphorylated by Shaggy. Recently, an effect of LiCl treatment was observed on axonal transport defects resulting from human Tau overexpression in Drosophila larvae, suggesting a functional interaction between Shaggy and Tau (Mudher et al., 2004). Here, we demonstrated that the protein Futsch was required for the overgrowth phenotype observed in sgg loss-of-function conditions.

How is the activity of Sgg controlled at the NMJ? The kinase Sgg is known to be inhibited in both the insulin and the WNT/
Wingless (Wg) signaling pathways (Cohen and Frame, 2001). There is little evidence of the presence of insulin-like peptides or insulin receptor-like proteins at the NMJ in Drosophila (Gorczyca et al., 1993). Wg has been shown to be released by larval motoneurons in Drosophila and to control the NMJ growth (Gramates and Budnik, 1999; Packard et al., 2002). The control of Sgg activity via different signaling pathways at the NMJ is still an open question.

\section{References}

Bourouis M (2002) Targeted increase in shaggy activity levels blocks wingless signaling. Genesis 34:99-102.

Broadie KS, Richmond JE (2002) Establishing and sculpting the synapse in Drosophila and C. elegans. Curr Opin Neurobiol 12:491-498.

Budnik V, Zhong Y, Wu CF (1990) Morphological plasticity of motor axons in Drosophila mutants with altered excitability. J Neurosci 10:3754-3768.

Cohen P, Frame S (2001) The renaissance of GSK3. Nat Rev Mol Cell Biol 2:769-776.

Gorczyca M, Augart C, Budnik V (1993) Insulin-like receptor and insulinlike peptide are localized at neuromuscular junctions in Drosophila. J Neurosci 13:3692-3704.

Gramates LS, Budnik V (1999) Assembly and maturation of the Drosophila larval neuromuscular junction. Int Rev Neurobiol 43:93-117.

Hall AC, Lucas FR, Salinas PC (2000) Axonal remodeling and synaptic differentiation in the cerebellum is regulated by WNT-7a signaling. Cell 100:525-535.

Klagges BR, Heimbeck G, Godenschwege TA, Hofbauer A, Pflugfelder GO, Reifegerste R, Reisch D, Schaupp M, Buchner S, Buchner E (1996) Invertebrate synapsins: a single gene codes for several isoforms in Drosophila. J Neurosci 16:3154-3165.

Lahey T, Gorczyca M, Jia XX, Budnik V (1994) The Drosophila tumor suppressor gene dlg is required for normal synaptic bouton structure. Neuron 13:823-835.

Lnenicka GA, Keshishian H (2000) Identified motor terminals in Drosophila larvae show distinct differences in morphology and physiology. J Neurobiol 43:186-197.

Lucas FR, Salinas PC (1997) WNT-7a induces axonal remodeling and increases synapsin I levels in cerebellar neurons. Dev Biol 192:31-44.

Morin X, Daneman R, Zavortink M, Chia W (2001) A protein trap strategy to detect GFP-tagged proteins expressed from their endogenous loci in Drosophila. Proc Natl Acad Sci USA 98:15050-15055.

Mudher A, Shepherd D, Newman TA, Mildren P, Jukes JP, Squire A, Mears A, Berg S, MacKay D, Asuni AA, Bhat R, Lovestone S (2004) GSK-3beta inhibition reverses axonal transport defects and behavioural phenotypes in Drosophila. Mol Psychiatry 9:522-530.

Muller D, Nikonenko I, Jourdain P, Alberi S (2002) LTP, memory and structural plasticity. Curr Mol Med 2:605-611.

Packard M, Koo ES, Gorczyca M, Sharpe J, Cumberledge S, Budnik V (2002) The Drosophila Wnt, wingless, provides an essential signal for pre- and postsynaptic differentiation. Cell 111:319-330.

Roos J, Hummel T, Ng N, Klambt C, Davis GW (2000) Drosophila Futsch regulates synaptic microtubule organization and is necessary for synaptic growth. Neuron 26:371-382.

Ruel L, Pantesco V, Lutz Y, Simpson P, Bourouis M (1993) Functional significance of a family of protein kinases encoded at the shaggy locus in Drosophila. EMBO J 12:1657-1669.

Schuster CM, Davis GW, Fetter RD, Goodman CS (1996a) Genetic dissection of structural and functional components of synaptic plasticity. I. Fasciclin II controls synaptic stabilization and growth. Neuron 17:641-654.

Schuster CM, Davis GW, Fetter RD, Goodman CS (1996b) Genetic dissection of structural and functional components of synaptic plasticity. II. Fasciclin II controls presynaptic structural plasticity. Neuron 17:655-667.

Torroja L, Packard M, Gorczyca M, White K, Budnik V (1999) The Drosophila beta-amyloid precursor protein homolog promotes synapse differentiation at the neuromuscular junction. J Neurosci 19:7793-7803. 